\title{
GeV Observations of the Extended Pulsar Wind Nebulae Constrain the Pulsar Interpretations of the Cosmic-Ray Positron Excess
}

\author{
Shao-Qiang Xi ${ }^{1,2}$, Ruo-Yu Liu ${ }^{3}$, Zhi-Qiu Huang ${ }^{1,2}$, Kun Fang $^{4}$, and Xiang-Yu Wang ${ }^{1,2}$ \\ ${ }^{1}$ School of Astronomy and Space Science, Nanjing University, Nanjing 210023, People's Republic of China; xywang@nju.edu.cn \\ ${ }^{2}$ Key Laboratory of Particle Astrophysics, Institute of High Energy Physics, Chinese Academy of Sciences, Beijing 100049, People's Republic of China \\ ${ }^{3}$ Deutsches Elektronen-Synchrotron (DESY), Platanenallee 6, D-15738 Zeuthen, Germany; ruoyu.liu@desy.de \\ ${ }^{4}$ Key laboratory of Modern Astronomy and Astrophysics, Nanjing University, Ministry of Education, Nanjing 210093, People's Republic of China \\ Received 2019 April 14; revised 2019 April 28; accepted 2019 May 8; published 2019 June 19
}

\begin{abstract}
It has long been suggested that nearby pulsars within $\sim 1 \mathrm{kpc}$ are the leading candidate of the $10-500 \mathrm{GeV}$ cosmicray positron excess measured by PAMELA and other experiments. The recent measurement of the surface brightness profile of $\mathrm{TeV}$ nebulae surrounding Geminga and PSR B0656+14 by the High-Altitude Water Cherenkov Observatory (HAWC) suggests inefficient diffusion of particles from the sources, giving rise to a debate on the pulsar interpretation of the cosmic-ray positron excess. Here we argue that $\mathrm{GeV}$ observations provide more direct constraints on the positron density in the $\mathrm{TeV}$ nebulae in the energy range of $10-500 \mathrm{GeV}$ and hence on the origin of the observed positron excess. Motivated by this, we search for $\mathrm{GeV}$ emission from the $\mathrm{TeV}$ nebulae with the Fermi Large Area Telescope (LAT). No spatially extended GeV emission is detected from these two $\mathrm{TeV}$ nebulae in the framework of two-zone diffusion spatial templates, suggesting a relatively low density of $\mathrm{GeV}$ electrons/positrons in the TeV nebulae. A joint modeling of the data from HAWC and Fermi-LAT disfavors Geminga and PSR B0656+14 as the dominant sources of the positron excess at $\sim 50-500 \mathrm{GeV}$ for the usual Kolmogorov-type diffusion, while for an energy-independent diffusion, a dominant part of the positron excess contributed by them cannot be ruled out by the current data.
\end{abstract}

Key words: cosmic rays

\section{Introduction}

Measurements of the cosmic-ray positron fraction by PAMELA and other experiments have found an excess in the energy range of $\sim 10-500 \mathrm{GeV}$ (Adriani et al. 2009; Ackermann et al. 2012c; Aguilar et al. 2013), relative to the standard predictions for secondary production in the interstellar medium (ISM). These high-energy positrons should be produced by nearby sources within $\sim \mathrm{kpc}$ due to severe radiative energy losses in the propagation to Earth (e.g., Aharonian et al. 1995). The origin of these positrons is still unknown, and the highly suggested candidate sources include nearby pulsars (e.g., Hooper et al. 2009; Yuskel et al. 2009; Profumo 2011; Yin et al. 2013; Joshi \& Razzaque 2017) and annihilating dark matter particles (e.g., Bergström et al. 2009; Yin et al. 2009; Cholis \& Hooper 2013; Silk 2014). Geminga and PSR B0656+14 are particularly attractive candidates for $\sim 50-500 \mathrm{GeV}$ positrons due to their proximity to Earth and their suitable ages.

Observations from Milagro (Abdo et al. 2009), along with recent observations by High-Altitude Water Cherenkov Observatory (HAWC; Abeysekara et al. 2017b), have revealed extended $\mathrm{TeV}$ emissions surrounding Geminga and PSR B0656+14. The angular sizes of the extended $\mathrm{TeV}$ nebulae are much larger than the X-ray PWNe. The intensity of the $\mathrm{TeV}$ emission indicates that these pulsars deposit a significant fraction of their total spin-down power into high-energy electrons and positrons (Hooper et al. 2017). Recently, HAWC measured the angular surface brightness profile (SBP) of the $\mathrm{TeV}$ nebulae around Geminga and PSR B0656+14 (Abeysekara et al. 2017a). The angular SBP suggests that the diffusion coefficient within at least a few tens of $\mathrm{pc}$ of these two pulsars is significantly lower than that in the normal ISM. The HAWC collaboration argues that such a lowdiffusion coefficient leads to a negligible positron flux at Earth, disfavoring them as the sources of the observed positron excess (Abeysekara et al. 2017a). However, it was later argued that such a low-diffusion region should be only restricted to a small region close to these pulsars (Hooper \& Linden 2018). By invoking a normal diffusion coefficient in the region outside the $\mathrm{TeV}$ nebula, the positron flux at Earth can be significantly increased (Fang et al. 2018; Profumo et al. 2018). Based on the above arguments, several works proposed that Geminga and PSR B0656+14 remain the best candidates for producing a dominant fraction of the observed positron excess at $\sim 50-500 \mathrm{GeV}$ (Fang et al. 2018; Hooper \& Linden 2018; Profumo et al. 2018).

The observed $\mathrm{TeV}$ gamma-rays are produced by electrons/ positrons with energy above $10 \mathrm{TeV}$ that up-scatter cosmic microwave background (CMB) photons, according to an approximate relation $E_{\gamma}=20 \mathrm{TeV}\left(E_{e} / 100 \mathrm{TeV}\right)^{2}$, where $E_{e}$ is the energy of electrons/positrons. Thus, $\mathrm{TeV}$ emission does not directly reflect the properties of positrons in the energy range of $10-1000 \mathrm{GeV}$ relevant to the measured cosmic-ray positron excess. Instead, these positrons should produce $\mathrm{GeV}$ emission through the inverse-Compton scattering of infrared and optical background photons. If we ascribe the measured positron excess to Geminga and PSR B0656+14, the required amount of injected electrons/positrons at $50-500 \mathrm{GeV}$ should be able to produce $\mathrm{GeV}$ nebulae around these pulsars. Motivated by this, we first study possible $\mathrm{GeV}$ emission associated with the $\mathrm{TeV}$ nebulae using the data from the Fermi Large Area Telescope (LAT) in Section 2. Then in Section 3, we study whether Geminga and PSR B0656+14 can be the dominant sources of the positron excess under the $\mathrm{GeV}$ constraints. We discuss the influence of various parameters of the diffusion models on the positron flux in Section 4. Finally, we give a summary in Section 5. 

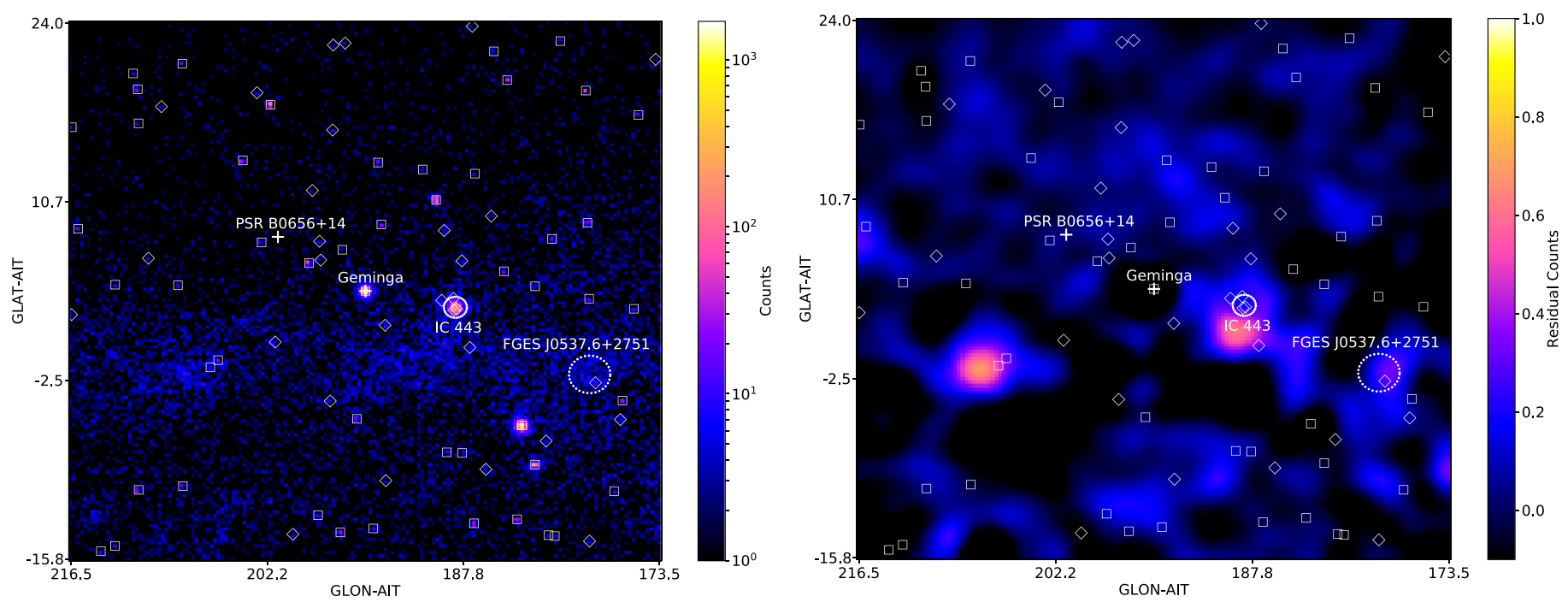

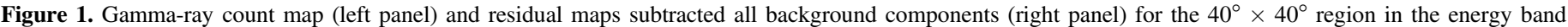

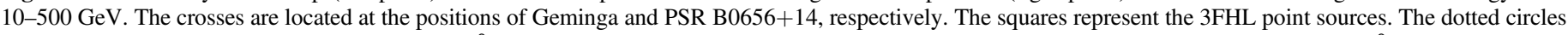

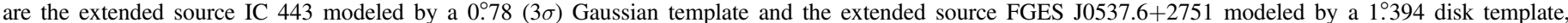

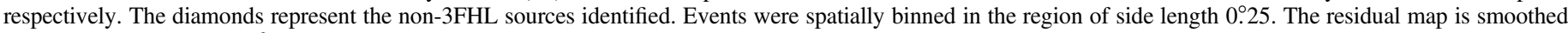
by Gaussian kernel $\left(\sigma=1^{\circ}\right)$.

\section{Analysis of Fermi-LAT Data}

Previous searches for the $\mathrm{GeV}$ emission around Geminga with Fermi-LAT have yielded non-detections (Ackermann et al. 2011). We here analyze the 10-yr Fermi-LAT data near the region of Geminga and PSR B0656+14, searching for possible spatially extended emission associated with the $\mathrm{TeV}$ nebulae.

\subsection{Count Map}

Employing the newest released Fermi Science Tools package (v11r5p3) with the instrument response functions (IRFs) P8R3_SOURCE_V2, we study the extended gamma-ray emission around Geminga and PRS B0656+14 using $10 \mathrm{yr}$ (from 2008 August 4 to 2018 September 17) of Pass 8 SOURCE data at energies between $10 \mathrm{GeV}$ and $500 \mathrm{GeV}$. We consider the photons within a $40^{\circ} \times 40^{\circ}$ region of interest (ROI) centered at positions $(l, b)=\left(195^{\circ} .14,4^{\circ} .27\right)$. Photons detected at zenith angles larger than $105^{\circ}$ were excised to limit the contamination from gamma-rays generated by cosmic-ray interactions in the upper layers of the atmosphere, and were further filtered by the relational filter expression (DATA QUAL $>0) \& \&$ (LAT_CONFIG $==1$ ) in gtmktime tool. We binned our data in 12 logarithmically spaced bins in energy and use a spatial binning of 0.05 per pixel.

The background model in this analysis includes: (1) the diffuse Galactic interstellar emission (IEM) template (gll_iem_v6.fits) released and described by the Fermi-LAT collaboration through the Fermi Science Support Center (Acero et al. 2016b), (2) the isotropic diffuse component accounting for the extragalactic diffuse gamma-ray background and misclassified cosmic rays (CRs) with a spectral shape described by iso_P8R3_SOURCE_V2.txt and (3) the point and extended sources listed in the third Catalog of Hard Fermi-LAT Sources (3FHL; Ajello et al. 2017). In addition, we search for new gamma-ray point sources using the gttsmap tool, and 30 non-3FHL sources are found, which are included in our background model. The counts map with the point and extended sources of the background model is shown in the left panel of Figure 1. We first perform the background-only fitting. All of the spectral parameters of the point and extended sources of the background model are left free. We also left three degrees of freedom to the diffuse emission, i.e., the normalizations of the isotropic and Galactic components, and a spectral index $\Gamma$ that can make the Galactic component a little harder or softer after it is multiplied by $\left(E / E_{0}\right)^{\Gamma}$. Based on the best-fit value from the background-only model fit, we obtain the residual map shown in the right panel of Figure 1. We do not find any obvious excess around Geminga and PSR B0656+14 in the residual map.

\subsection{Two-zone Diffusion Templates for Fermi-LAT Data Analysis}

It has been argued that the assumption of a low-diffusion coefficient in the local region is inconsistent with the detection of the highest-energy (up to $20 \mathrm{TeV}$ ) electrons by High Energy Stereoscopic System (H.E.S.S.; Hooper \& Linden 2018). Thus, it is more reasonable to consider that the low-diffusion region is only restricted to the region close to pulsars and the outer region has a normal diffusion coefficient, as already suggested in Fang et al. (2018) and Profumo et al. (2018). In addition, possible mechanisms for the suppression of the diffusion coefficient in the region close to pulsars have been suggested (Evoli et al. 2018). Following this, we consider a two-zone model for electron diffusion and adopt a step function for the diffusion coefficient

$$
D\left(E_{e}, r\right)= \begin{cases}D_{1}, & r<r_{0} \\ D_{2}, & r \geqslant r_{0}\end{cases}
$$

Following Abeysekara et al. (2017a), $\quad D_{1}=4.5 \times$ $10^{27}\left(\frac{E_{e}}{100 \mathrm{TeV}}\right)^{1 / 3} \mathrm{~cm}^{2} \mathrm{~s}^{-1}$ is considered for the vicinity of the pulsar, where the Kolmogorov-type diffusion (i.e., with energy dependence $\delta=1 / 3$ ) is assumed for the inner region. For the diffusion coefficient in the ISM, we take the GALPROP default 
value, $D_{2}=1.8 \times 10^{30}\left(\frac{E_{e}}{100 \mathrm{TeV}}\right)^{1 / 3} \mathrm{~cm}^{2} \mathrm{~s}^{-1}$, as inferred from measurements of the boron-to-carbon ratio and other cosmicray secondary-to-primary ratios (Hooper \& Linden 2018; Profumo et al. 2018). For convenience, we call such a combination of parameter values (i.e., $r_{0}=50 \mathrm{pc}$, $D_{1}=4.5 \times 10^{27}\left(\frac{E_{e}}{100 \mathrm{TeV}}\right)^{1 / 3} \mathrm{~cm}^{2} \mathrm{~s}^{-1}, \quad B_{1}=3 \mu \mathrm{G}, \quad p=2.25$ and $D_{2}=1.8 \times 10^{30}\left(\frac{E_{e}}{100 \mathrm{TeV}}\right)^{1 / 3} \mathrm{~cm}^{2} \mathrm{~s}^{-1}$ ) the benchmark case. Hooper et al. (2017) has shown that, even if all pulsars have such a low-diffusion halo, there is little impact on the observed secondary-to-primary ratios (e.g., the boron-to-carbon ratio) in the cosmic-ray spectrum as measured at Earth as long as $r_{0} \leqslant 50 \mathrm{pc}$. This is because such low-diffusion regions occupy only a fraction of $<3 \%$ of the volume of the Milky Ways disk (Hooper et al. 2017).

\subsection{Flux Limits and TS Values for Various Spatial Templates}

We evaluate the test statistic (TS) for an additional extended source, defined as TS $=-2\left(\ln L_{0}-\ln L\right)$, where $L_{0}$ is the maximum-likelihood value for the null hypothesis and $L$ is the maximum likelihood with the additional extended source described by the templates. First, we create a $2^{\circ}$ disk template, naively assuming that $\mathrm{GeV}$ emission has the same size as that of the $\mathrm{TeV}$ halo in the HAWC catalog (Abeysekara et al. 2017b). Then, we consider a one-zone diffusion template (named Diffusion 1 template hereafter), assuming that $\mathrm{GeV}$ emission follows that predicted by the one-zone diffusion model adopting the same parameters as used in Abeysekara et al. (2017a).

Next, we focus on the two-zone diffusion models. We first build a two-zone template considering an inner region as that for the $\mathrm{TeV}$ halo and an outer region assuming a normal diffusion coefficient in the ISM. We name this template the Diffusion 2 template.

Since the GeV gamma-ray flux overshoots the upper limits of Fermi-LAT for the Diffusion 2 template (see Section 3.2 for details), we adjust the electron spectral index from soft to hard and thus obtain the Diffusion 3 template for which the expected flux just touches the upper limit. In addition, on the premise of fitting the flux and the SBP of the TeV nebulae measured by HAWC, we create two more extended source templates: i.e., the Diffusion 4 template corresponds to the case changing $r_{0}=50-100 \mathrm{pc}$, and the Diffusion 5 template corresponds to the case changing the energy dependence of the diffusion coefficient of the inner region to $\delta=0$. For the $\mathrm{GeV}$ emission around PSR B0656+14, we simply consider the two-zone diffusion models for a Kolmogorov-type diffusion of inner region (Diffusion 6) and for an energy-independent diffusion of inner region (Diffusion 7). Note that we relocate the ROI center at the position of the PSR B0656+14 when fitting the Diffusion 6 and Diffusion 7 templates.

We obtain the radial SBPs by integrating the gamma-ray flux over the line of sight for a given angle $\theta$ from the location of the pulsar. The obtained intensity as a function of $\theta$, i.e., $I(\epsilon, \theta)$ is used as the spatial template for analyzing the Fermi-LAT data. The SBPs at 10 and $500 \mathrm{GeV}$ are shown as examples in Figure 2. The angular extension for the $\mathrm{GeV}$ emission is much larger than that of the $\mathrm{TeV}$ emission since the $\mathrm{GeV}$-emitting electrons can travel a larger distance before losing their energy than TeV-emitting electrons, especially in the one-diffusion-

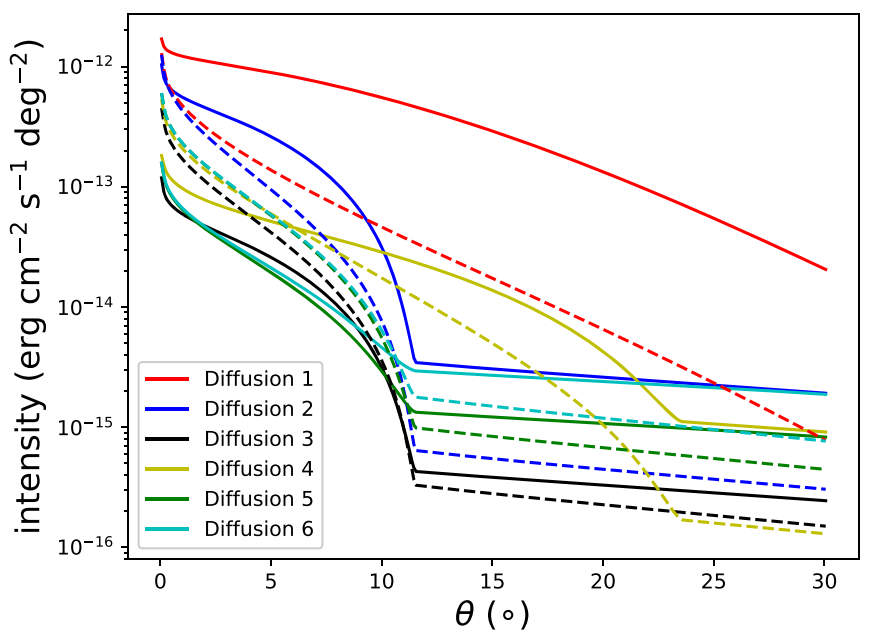

Figure 2. The surface brightness profiles at $10 \mathrm{GeV}$ (solid line) and at $500 \mathrm{GeV}$ (dashed line) for each diffusion model listed in Table 1.

zone model. In the presence of two diffusion zones, the negative gradient of electron density is large near the boundary between the two diffusion zones, leading to a quick drop of the surface brightness with distance to the pulsar.

Since the expected spatial distributions of the $\mathrm{GeV}$ emission are energy dependent for the above diffusion models, we create the templates using the mapcube file, which is a threedimensional FITS map allowing arbitrary spectral variation as a function of sky position. We cut the templates at $25^{\circ}$, within which more than $90 \%$ of the integrated flux lies for all the above diffusion templates. Note that the spectral parameters of the background sources are still left free for the analysis of each template.

No significant extended $\mathrm{GeV}$ emission is detected from the $\mathrm{TeV}$ nebulae of Geminga and PSR B0656+14. The flux limits for various spatial templates are obtained and are shown in Table 1. Spatial templates with larger angular extension usually result in larger TS values. The large difference in the limits for different templates is due to the large difference in the sizes and angular profiles of these templates.

To assess the robustness of our results listed in Table 1, we perform a number of systematic checks such as varying the ROI size and selecting different event class data. In particular, we investigate how the diffuse Galactic emission modeling affects the TS values and the upper limits using eight alternative IEM templates (Ackermann et al. 2012a; Acero et al. 2016a). The details of these eight alternative IEM templates are given in the Appendix. The total systematic uncertainties of the upper limits for the diffusion templates are about 32\% (see Table 2 in the Appendix).

\section{Implications for the Positron Flux at Earth}

Below we argue that the flux limits of gamma-rays in $10-500 \mathrm{GeV}$ can impose meaningful constraints on the positron flux nearby the pulsars and hence on their contribution to the positron flux observed at Earth.

\subsection{The Electron/Positron Flux Calculation}

To obtain the theoretical $\mathrm{GeV}$ emission from the $\mathrm{TeV}$ nebulae, we first need to calculate the distribution of electrons / positrons, which can be obtained through the following 
Table 1

Statistic Test and 95\% Upper Limit Fluxes for Each Extended Template

\begin{tabular}{lcccc}
\hline \hline $\begin{array}{l}\text { Model Name } \\
\text { (Geminga) }\end{array}$ & $\begin{array}{c}\text { UL }(10-500 \mathrm{GeV}) \\
\times 10^{-10} \mathrm{ph} \mathrm{cm}^{-2} \mathrm{~s}^{-1}\end{array}$ & TS & $\begin{array}{c}\text { Model Name } \\
\text { (PSR B0656+14) }\end{array}$ & $\begin{array}{c}\text { UL }(10-500 \mathrm{GeV}) \\
\times 10^{-10} \mathrm{ph}^{-2} \mathrm{~s}^{-1}\end{array}$ \\
\hline $2^{\circ}$ Disk & 0.44 & 0.1 & $2^{\circ}$ Disk & 0.61 \\
Diffusion 1 & 29.1 & 16.1 & Diffusion 6 & 2.8 \\
Diffusion 2 & 6.3 & 4.1 & Diffusion 7 & 0.9 \\
Diffusion 3 & 4.5 & 2.1 & & 0.5 \\
Diffusion 4 & 14.3 & 6.6 & & 0.5 \\
Diffusion 5 & 3.9 & 1.0 & & \\
\hline
\end{tabular}

Note. The upper-limit fluxes in 10-500 GeV at the 95\% confident level. The meaning of the model names for Geminga are as follows: (1) Disk represents an uniform disk with a radius of $2^{\circ}$ similar to that in the HAWC catalog (Abeysekara et al. 2017b); (2) Diffusion 1 template corresponds to the Fermi-LAT's gamma-ray profiles predicted by the one-zone model adopting the same parameters as in Abeysekara et al. (2017a; see the left panel of Figure 1); (3) Diffusion 2-5 templates correspond to two-zone models with the parameters the same as that used in the Figures 3-7, respectively. The meaning of the model names for PSR B0656+14 are as follows: (1) Disk represents an uniform disk with a radius of $2^{\circ}$ similar to that in the HAWC catalog (Abeysekara et al. 2017b); (2) The Diffusion 6 and Diffusion 7 templates correspond to the two-zone Diffusion model for a Kolmogorov-type diffusion and an energy-independent diffusion in the inner region, respectively. Note that all the Diffusion templates are cut at $25^{\circ}$.

transport equation, assuming the pulsar is located at $r=0$ :

$$
\begin{aligned}
\frac{\partial N(\gamma, r, t)}{\partial t}= & \frac{1}{r^{2}} \frac{\partial}{\partial r}\left(r^{2} D(\gamma, r) \frac{\partial N}{\partial r}\right) \\
& -\frac{\partial}{\partial \gamma}(\dot{\gamma} N)+Q(\gamma, t) \delta(r),
\end{aligned}
$$

where $N(\gamma, r, t)$ represents the density of electrons/positrons with Lorentz factor $\gamma$ at the spacetime of $(r, t), D(\gamma, r)$ is the diffusion coefficient at a distance $r$ to the pulsar, $\dot{\gamma}$ is the cooling rate of electrons/positrons due to both synchrotron radiation and inverse Compton radiation, and $\delta(r)$ is the Dirac delta function, which signifies electron/positron injection only from the pulsar. The cooling rate, taking into account the Klein-Nishina effects, can be approximately given by (Moderski et al. 2005)

$$
\dot{\gamma}=\frac{4 \sigma_{T} \gamma^{2}}{m_{e} c}\left[U_{B}+\sum_{i} \frac{U_{\mathrm{ph}, i}}{\left(1+4 \gamma \frac{\epsilon_{T, i}}{m_{e} c^{2}}\right)^{3 / 2}}\right],
$$

where $U_{B}$ is the magnetic field energy density and $U_{\mathrm{ph}, i}$ represents the radiation field energy density, including CMB, infrared, and optical photons. The photon fields are considered to have a graybody distribution, for which the temperature and energy density are approximately those derived by GALPROP (Moskalenko \& Strong 1998; Abeysekara et al. 2017a). $\epsilon_{T, i}$ is the average photon energy of the radiation field, which is equal to $2.82 \mathrm{kT}$ in the case of blackbody or graybody radiation with $k$ being the Boltzmann constant and $T$ being the temperature. The injection term is assumed to be $Q(\gamma, t)=J(\gamma) S(t)$, where $J(\gamma) \propto \gamma^{-p} e^{-\gamma / \gamma_{\max }}$ with $p$ being the spectral index and $\gamma_{\max }$ being the maximum Lorentz factor of electrons injected by the pulsar, and $S(t) \propto 1 /(1+t / \tau)^{2}$ assuming that the pulsar is a pure dipole radiator of a braking index of 3 and the spin-down timescale of the pulsar $\tau=12000 \mathrm{yr}$. The normalization of the injection term can be found by $\iint Q(\gamma, t) \gamma m_{e} c^{2} d \gamma d t=W_{e}$, where $W_{e}$ is the total injection power of electrons and positrons. The numbers of injected electrons and positrons are the same at any energy. We solve the equation by discretizing the equation with a numerical method similar to that employed in Fang et al. (2018).

\subsection{One-zone Diffusion Scenario}

First, we reproduce the gamma-ray flux and the SBP in 8-40 $\mathrm{TeV}$ of the TeV nebula of Geminga measured by HAWC, ${ }^{5}$ by adopting the same parameters used in Abeysekara et al. (2017a), which assumes that the inefficient diffusion region inferred from $\mathrm{TeV}$ nebulae extends all the way to Earth. As we can see from the left panel of Figure 3, the contribution to the positron flux by Geminga is very low due to the very lowdiffusion coefficient, consistent with the result in Abeysekara et al. (2017a). However, while HAWC's measurement is well fitted, the expected $\mathrm{GeV}$ fluxes exceed the upper limits of Fermi-LAT by about one order of magnitude. This demonstrates the importance of considering the $\mathrm{GeV}$ observations consistently. By invoking a hard electron spectrum, one can in principle get a consistently low GeV flux. Since our work focuses on the two-zone diffusion scenarios, we will not discuss this in detail.

\subsection{Two-zone Diffusion Scenario}

As shown in Figure 4, the multi-TeV spectrum and the SBP can be fitted with most parameters remaining unchanged except introducing an additional outer fast-diffusion region at $r>r_{0}=50 \mathrm{pc}$, while the predicted positron flux at Earth is significantly increased, accounting for $70 \%$ of the measured positron flux above $100 \mathrm{GeV}$. This is consistent with the conclusion in previous literature (Fang et al. 2018; Profumo et al. 2018; Tang \& Piran 2019). However, the expected GeV gamma-ray flux also overshoots the upper limits of Fermi-LAT by more than one order of magnitude. This demonstrates the important role of the $\mathrm{GeV}$ observations in constraining the positron flux from pulsars.

On the premise of fitting the flux and the SBP of the TeV nebulae measured by HAWC, we need to reduce the ratio between multi-GeV gamma-ray flux and multi-TeV gamma-ray flux. As a result, a harder electron injection spectrum is required, or a spectral break needs to be introduced around $\mathrm{TeV}$ in order to reconcile the predicted multi-GeV flux with the observed flux by Fermi-LAT. We here adopt the former choice since the number of the free parameters in this case is less. Note that although Abeysekara et al. (2017a) suggests a spectral

\footnotetext{
5 Except that the injection electron/positron power $W_{e}$ in our calculation is about 0.5 times of that in Abeysekara et al. (2017a).
} 
Table 2

Systematic Uncertainties

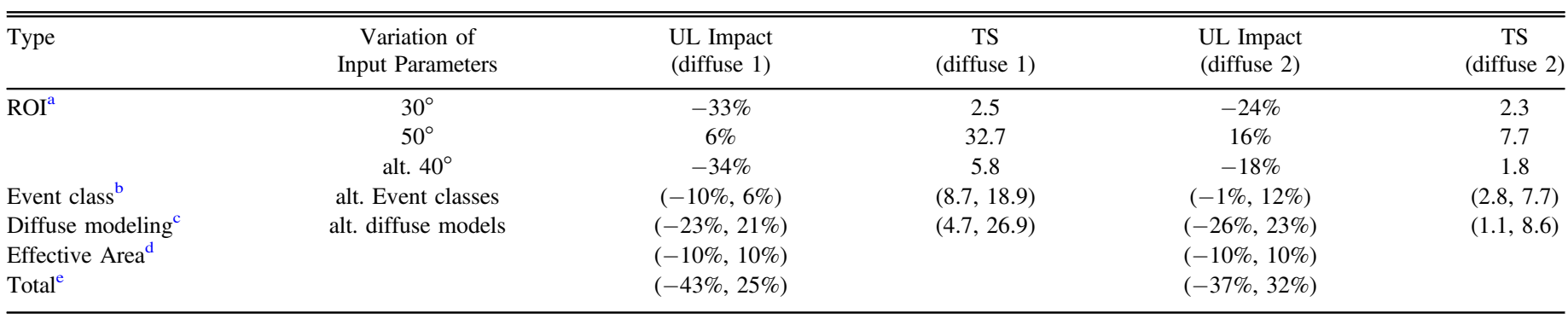

Notes. Overview of systematic uncertainties.

${ }^{\mathrm{a}}$ We select the ROI of $30^{\circ} \times 30^{\circ}, 50^{\circ} \times 50^{\circ}$ and alternative $40^{\circ} \times 40^{\circ}$ centered at the position of Geminga to repeat our analysis. The alt. $40^{\circ}$ represents $40^{\circ} \times 40^{\circ}$ square ROI that is parallel to R.A. and decl. Note that we get a lower TS value, $\sim 22$, in the analysis of ROI $50^{\circ} \times 50^{\circ}$ using the newest released IEM (gll_iem_v07. fits) and the background sources of the Fourth Fermi-LAT Source Catalog (4FGL).

$\mathrm{b}$ The CLEAN, UTRACLEAN, UTRACLEANVETO and SOURCEVETO conversion events are used to perform the analysis.

${ }^{\mathrm{c}}$ See the Table 3 for details.

d Generally, the uncertainties in the effective area are estimated by using modified IRFs whose effective area bracket that of our nominal IRF. These "biased" IRFs are defined by envelopes above and below the nominal dependence of the effective area with energy by linearly connecting differences of $(5 \%, 10 \%)$ at $\log (E)$ of $(4,5.2)$, respectively (Ackermann et al. 2012b). We simply consider the uncertainties of effective area for estimating upper limit as $\sim 10 \%$ in our analysis energy band.

${ }^{\mathrm{e}}$ We combine all the errors in quadrature to obtain our total systematic uncertainty.

index of $p \simeq 2.25$ to fit the multi-TeV spectrum, a harder electron injection spectrum in combination with a relatively small $\gamma_{\max }$ can lead to a similar spectral shape in the range of $8-40 \mathrm{TeV}$.

There are various sets of parameters that are able to fit the multi-TeV spectrum and the SBP, but the corresponding positron fluxes at Earth are different. We need to find out the maximally allowed positron flux at Earth produced by Geminga, and in the mean time take into account the observations of HAWC and Fermi-LAT. Given that the diffusion in the inner region generally dominates the propagation time of positrons from the pulsar to Earth, we require

$$
D_{1}(100 \mathrm{GeV}) \gtrsim r_{0}^{2} / 4 \tau_{\mathrm{Gem}}=5 \times 10^{26}\left(\frac{r_{0}}{50 \mathrm{pc}}\right)^{2} \mathrm{~cm}^{2} \mathrm{~s}^{-1},
$$

since otherwise the injected positrons have not yet arrived at Earth after a propagation time equal to the lifetime of Geminga $\left(\tau_{\mathrm{Gem}}=340 \mathrm{kyr}\right)$. On the other hand, if the diffusion coefficient is too large, the injected electrons/positrons will distribute in a very large volume, and hence the flux at Earth will also be low. Furthermore, given the fast drop in the HAWC measured SBP within $5^{\circ}$, the spatial extension of multi-TeV-emitting electrons/positrons should not significantly exceed $25 \mathrm{pc}$. Since the diffusion distance of these very high-energy electrons/ positrons is determined by the radiative loss, we find

$$
D_{1}(100 \mathrm{TeV}) \lesssim 3 \times 10^{27}\left[\left(\frac{B_{1}}{3 \mu \mathrm{G}}\right)^{2}+0.6\right] \mathrm{cm}^{2} \mathrm{~s}^{-1} .
$$

where $B_{1}$ is the magnetic field of the inner diffusion region and the coefficient 0.6 in the square bracket accounts for the IC cooling on CMB photons. For the considered Kolmogorov-type diffusion (i.e., $\left.D \propto E^{1 / 3}\right)$, we find that $D_{1}\left(E_{e}\right)=5 \times$ $10^{26}\left(E_{e} / 100 \mathrm{GeV}\right)^{1 / 3} \mathrm{~cm}^{2} \mathrm{~s}^{-1}$ satisfies the above two equations for $r_{0}=50 \mathrm{pc}$ and $B_{1}=3 \mu \mathrm{G}$. We show the expected gammaray spectrum, SBP, and positron flux at Earth for such a set of parameters in Figure 5. Such a set of parameters is very close to our benchmark case, except that a hard injection spectrum with $p=1.7$ (or harder) is needed in order not to overshoot the upper limits imposed by Fermi-LAT. The resulting maximum positron fluxes above $\sim 100 \mathrm{GeV}$ are less than $10 \%$ of the measured value by AMS-02 (Aguilar et al. 2014).

Based on Equation (4), we expect that the positron flux at Earth increases with the size of the inner inefficient diffusion region $r_{0}$ (see Figure 6). This is because a larger $r_{0}$ corresponds to a larger $D_{1}$ and consequently a faster diffusion of particles. As a result, it requires a larger electron/positron injection power (i.e., $W_{e}$ ) to fit the $\mathrm{TeV}$ gamma-ray flux, subsequently leading to a higher positron flux at Earth. However, a larger $D_{1}$ also results in a flatter SBP. To make the predicted SBP as steep as the measured one, we need to invoke a relatively high magnetic field $\left(B_{1}\right)$ for the inner region as indicated by Equation (5). We now consider a large size for the inner zone with $r_{0}=100 \mathrm{pc}$, requiring $D_{1}(100 \mathrm{GeV}) \gtrsim 2 \times 10^{28} \mathrm{~cm}^{2} \mathrm{~s}^{-1}$ according to Equation (4). The magnetic field of the inner region $B_{1}>8 \mu \mathrm{G}$ should also be invoked according to Equation (5). The required magnetic field is larger than the typical ISM magnetic field (i.e., 3-6 $\mu \mathrm{G}$ ), and together with the large $r_{0}$ leads to a magnetic field energy in the inner region that is too large, i.e., $W_{B, 1}=B_{1}^{2} r_{0}^{3} / 6 \simeq 3 \times 10^{50} \mathrm{erg}$, which far exceeds the total energy of injected electrons/positrons from pulsar spin-down and is comparable to the total energy released in a supernova explosion. One has to seek a very efficient mechanism for amplification of the magnetic field with the energy being provided by some extra sources other than pulsars, or alternatively argue that the high magnetic field somehow originally pre-existed in this region. Either of the two choices requires extreme conditions.

\section{Influence of Some Parameters}

In the above text, we have already discussed the influence of $r_{0}, D_{1}$, and $B_{1}$ on the result. We here discuss the influence of the energy dependence of $D_{1}\left(E_{e}\right)$ (i.e., $\delta$ ) and the spin-down timescale of Geminga $\tau$. 

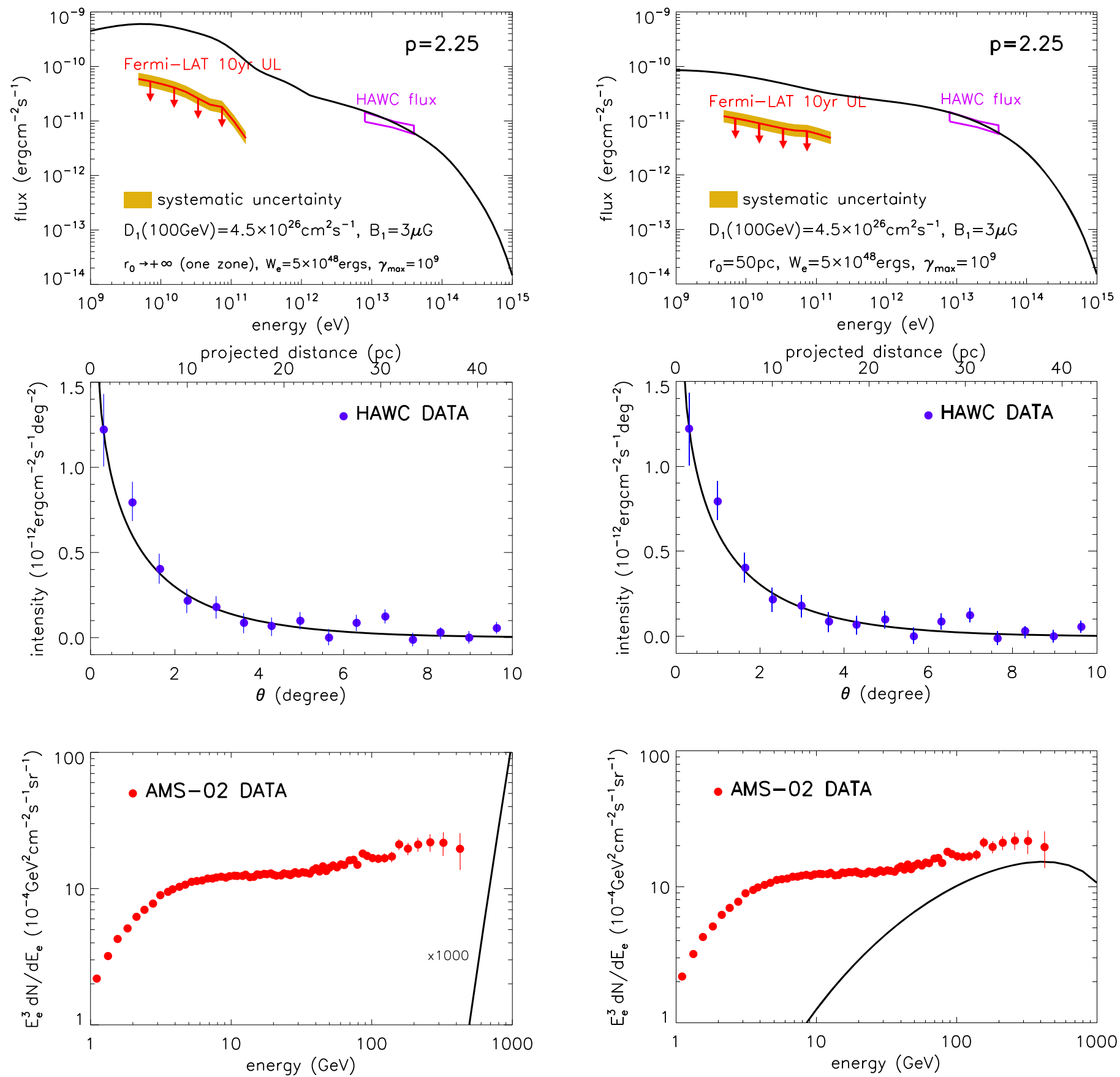

Figure 3. Comparison between observational data and theoretical expectation in the one-zone diffusion model for the gamma-ray spectrum within $25^{\circ}$ of Geminga (top panel), the surface brightness profile of $8-40 \mathrm{TeV}$ emission (middle panel), and the positron flux at Earth (bottom panel). The GeV flux limits are obtained using the diffusion spatial templates in accordance with the respective theoretical models (see Table 1). The TeV spectral and SBP data measured by HAWC are taken from Abeysekara et al. (2017a). The positron flux data measured by AMS-02 are taken from Aguilar et al. (2014).

\subsection{Energy Dependence of the Diffusion Coefficient in the Inner Region $\mathrm{D}_{1}$}

If we relax the pre-assumption of the Kolmogorov-type diffusion coefficient for the inner region, we can obtain a relatively large $D(100 \mathrm{GeV})$ while keeping the value of $D(100 \mathrm{TeV})$ the same by assuming a weaker rigidity dependence (a smaller $\delta$ ) in the diffusion coefficient, which subsequently avoids the

Figure 4. Comparison between observational data and theoretical expectation in the two-zone diffusion model where a standard ISM diffusion is assumed for the outer zone beyond $r_{0}=50 \mathrm{pc}$.

requirement of a too large magnetic field. Let us take an energyindependent diffusion coefficient (i.e., $\delta=0$ ) for the inner region as an example. We normalize $D_{1}$ to $4.5 \times 10^{27} \mathrm{~cm}^{2} \mathrm{~s}^{-1}$ at $100 \mathrm{TeV}$, which is the best-fit diffusion coefficient from HAWC's observation. The energy-independence of $D_{1}$ increases the diffusion coefficient at $100 \mathrm{GeV}$ by one order of magnitude compared to the benchmark case. As a result, the density of GeVemitting electrons/positrons residing in the inner region is significantly reduced, and the resulting multi-GeV flux is reduced to a level consistent with the Fermi-LAT's upper limits (see Figure 7) for a softer electron index of $p=2.25$. On the other hand, the faster diffusion leads to a more extended spatial 

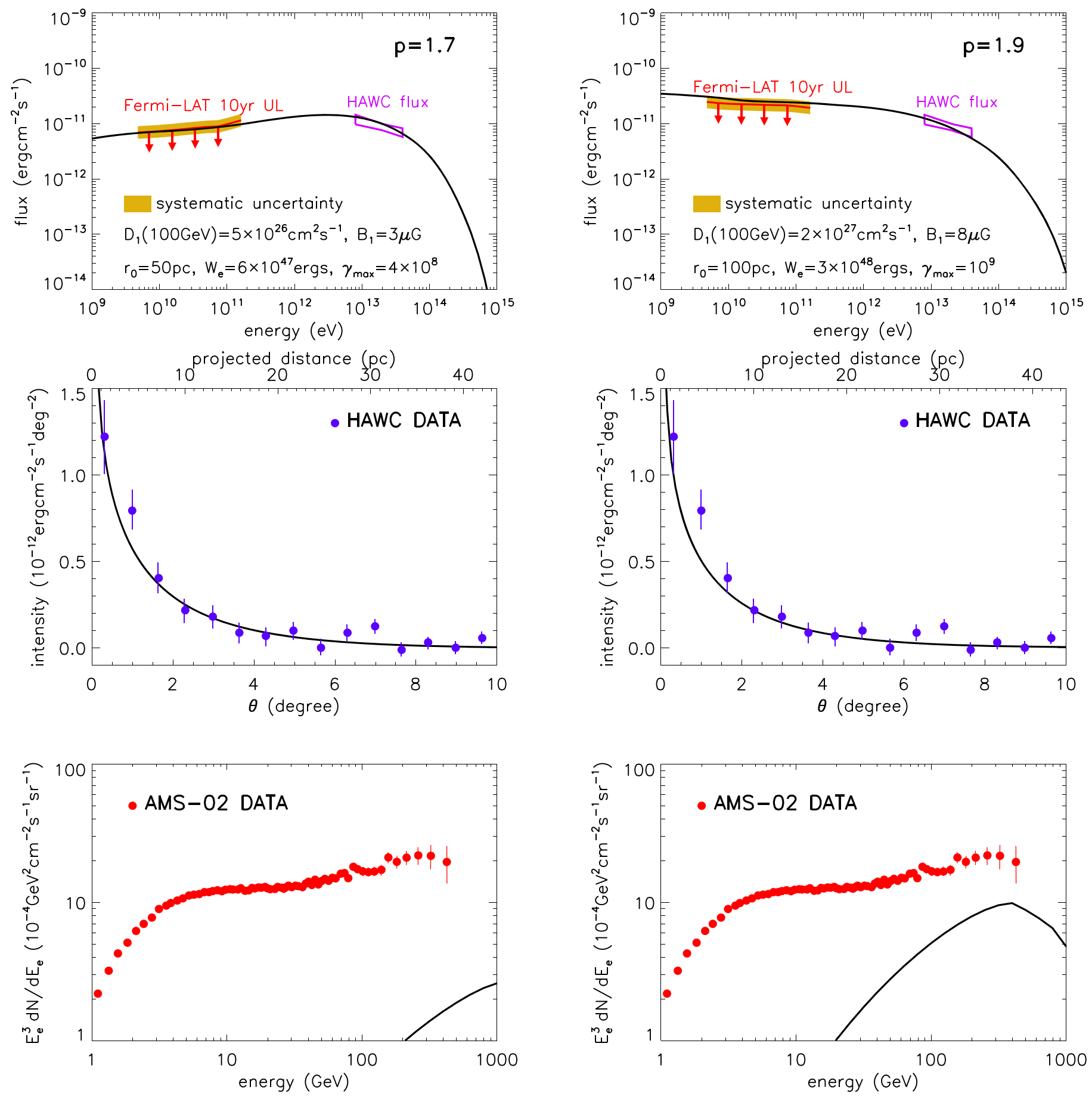

Figure 5. Same as Figure 4, but considering the constraint from the FermiLAT's observation.

distribution of $\sim 100 \mathrm{GeV}$ positrons, and the positron flux at Earth reaches a level of $30 \%$ of the measured flux above $100 \mathrm{GeV}$. In this case, if we further tune down the employed diffusion coefficient for the outer region $D_{2}$ by a factor of $2-3$, the positron flux can be increased to a level comparable to the measured flux.

For a more extended exploration on the influence of $\delta$, we fix $D_{1}(100 \mathrm{TeV})=4.5 \times 10^{27} \mathrm{~cm}^{2} \mathrm{~s}^{-1}$ and adjust $\delta$ to see the change. In Figure 8, we compare the expected gamma-ray fluxes (top panel), SBPs in $8-40 \mathrm{TeV}$ (middle panel) and the spatial distributions of $100 \mathrm{GeV}$ positron energy density (bottom panel) for $\delta=0,1 / 10,1 / 5,1 / 3,1 / 2$, and 1 . All the

Figure 6. Same as Figure 5, but assuming a larger boundary radius of $r_{0}=100 \mathrm{pc}$.

other parameters are the same as those in the benchmark case. We can see that while the flux and the SBP measured by HAWC are fitted, the GeV fluxes and the distributions of positron density with different $\delta$ vary with $\delta$ significantly. Except for the case of $\delta=0$, the expected $\mathrm{GeV}$ fluxes overshoot the Fermi-LAT's upper limits. This is understandable: since the diffusion coefficient at $100 \mathrm{TeV}$ is fixed, a larger $\delta$ results in a smaller diffusion coefficient at $100-1000 \mathrm{GeV}$, which is responsible for the multi-GeV flux. Consequently, many $\mathrm{GeV}$-emitting electrons/positrons reside in the vicinity of Geminga and give rise to relatively high $\mathrm{GeV}$ fluxes. We can 

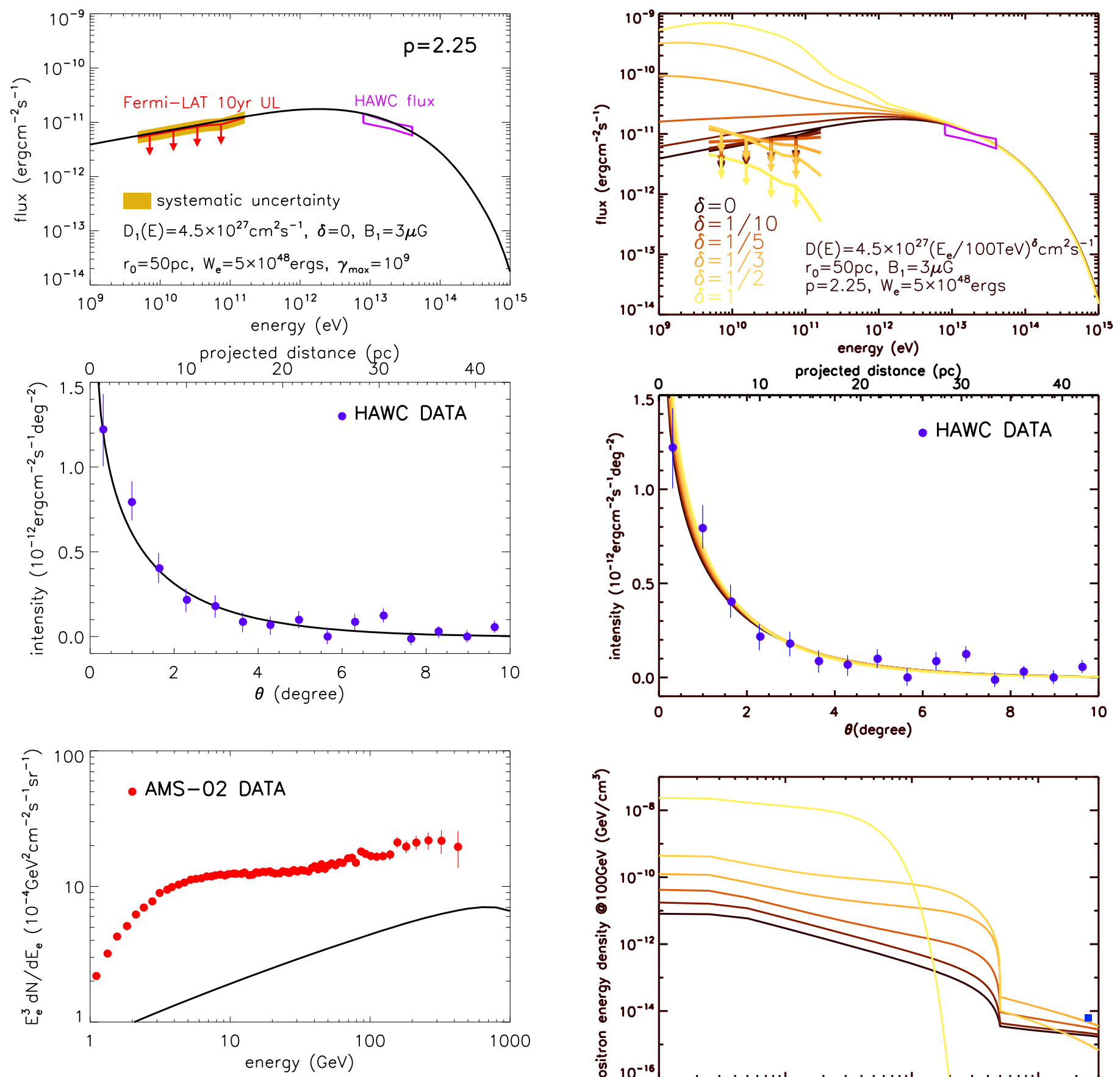

Figure 7. Results for the case assuming an energy-independent diffusion in the inner region around Geminga.

also see that $\delta=1 / 3$ results in the highest positron flux at $100 \mathrm{GeV}$ among all the considered $\delta$. It can be understood as follows: given $D_{1}(100 \mathrm{TeV})=4.5 \times 10^{27} \mathrm{~cm}^{2} \mathrm{~s}^{-1}$ and $\delta=$ $1 / 3$, the resulting diffusion coefficient for $100 \mathrm{GeV}$ electrons/ positrons satisfy the relation shown by Equation (4). A larger $\delta$ results in a smaller diffusion coefficient for $100 \mathrm{GeV}$ electrons / positrons so that the quantity that reaches Earth is low. On the contrary, a small $\delta$ results in a larger diffusion coefficient and makes the electrons/positrons distribute in a more extended volume, leading to a lower positron flux at Earth when the total injection is fixed. Lastly, we note that the energy-independent diffusion is quite different from the convection-dominated

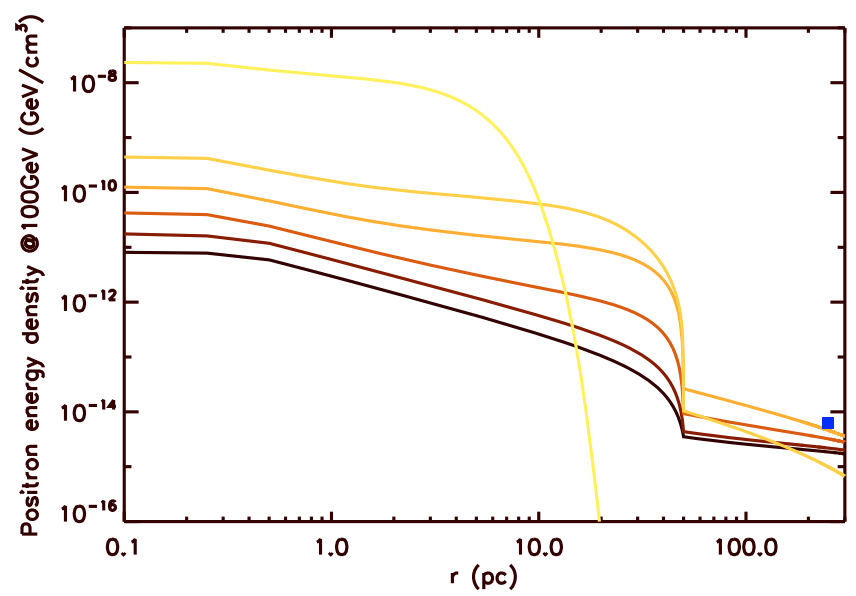

Figure 8. Results for the different energy dependence $(\delta)$ of the diffusion coefficient in the inner zone $D_{1}$. The diffusion coefficient is fixed to be $4.5 \times 10^{27} \mathrm{~cm}^{2} \mathrm{~s}^{-1}$ at $100 \mathrm{TeV}$, following the best-fit value obtained in HAWC's paper Abeysekara et al. (2017a). Other parameters are the same as those in the benchmark case. Top panel: gamma-ray flux from the region of a radius of $25^{\circ}$ centered at Geminga. Middle panel: $8-40 \mathrm{TeV}$ SBP. Bottom panel: positron energy density at $100 \mathrm{GeV}$ as a function of the distance to Geminga. Earth is located at $r=250 \mathrm{pc}$. The blue square shows the $100 \mathrm{GeV}$ positron energy density at Earth inferred from the AMS-02 measurements.

transportation. In the convection-dominated transportation model, the CR density would drop much faster than that in the diffusion model, so this model has difficulty in explaining the measured SBP. 


\subsection{The Spin-down Timescale $\tau$}

The spin-down timescale of a pulsar depends on the the surface magnetic field and the initial rotation period of the pulsar. In all the above calculations, we have taken $\tau=12000 \mathrm{yr}$ following Abeysekara et al. (2017a). However, the uncertainties in the above properties of Geminga can easily lead to a difference of a factor of a few in the spin-down timescale. Note that $\mathrm{TeV}$-emitting electrons/positrons cool quite fast with a cooling time of $10^{11} \mathrm{~s}$, which is only about $1 \%$ of the Geminga's age. Thus, the observed $\mathrm{TeV}$ emission is mainly contributed by electrons/positrons injected very recently. A larger spin-down timescale means a higher injection power at late time provided that the total injection energy is the same. Thus, we can use a smaller total injection power to fit HAWC's observation, and this would also decrease the multi-GeV gamma-ray flux and the $100 \mathrm{GeV}$ positron flux. In contrast, a smaller spin-down timescale leads to a smaller injection power at late time, and a larger total injection energy is needed to fit HAWC's observation. In this case, the multi-GeV flux and the $100 \mathrm{GeV}$ positron flux will be increased. In Figure 9, we show the results with $\tau=12000,24000,6000$, and $1000 \mathrm{yr}$, while other parameters are the same as those in the benchmark case. By comparing the results in the former three cases, we can see that the positron flux at Earth does not change much by varying $\tau$ by a factor of 2. Particularly, the positron flux in the case of $\tau=24000 \mathrm{yr}$ is quite low while the multi-GeV flux almost saturates the Fermi-LAT's upper limit, implying that the positron flux cannot be significantly increased by employing a softer injection spectrum. Based on this result, we can also expect that an even larger $\tau$ would only result in a lower positron flux. In accordance with our analysis above, $\tau=6000 \mathrm{yr}$ results in a positron flux that is slightly higher, but still much lower than the measured one. This motivates us to look into the result with an even smaller spin-down timescale of $\tau=1000 \mathrm{yr}$. As we can see, although the positron flux is enhanced with such a small $\tau$, the multi-GeV flux significantly exceeds Fermi-LAT's upper limit. We thus conclude that the uncertainty in the spin-down timescale will not affect our conclusion.

\subsection{The Contribution to the Positron Flux by PSR B0656+14}

PSR B0656+14 is another nearby pulsar that is suggested to be able to contribute significantly to the positron flux. The positron flux contributed by PSR B0656+14 can be calculated in the same way. The difference is the age of PSR B0656+14 $(110 \mathrm{kyr})$ and the distance to Earth $(288 \mathrm{pc})$. According to Equation (1), the maximum positron flux is obtained when $D_{1}(100 \mathrm{GeV})=1.7 \times 10^{27} \mathrm{~cm}^{2} \mathrm{~s}^{-1}$ for $r_{0}=50 \mathrm{pc}$. To fit the spectral and SBP data of PSR B0656+14 and simultaneously reconcile the upper limits imposed by Fermi-LAT, we find that a spectral index harder than $p=-1.9$ is required for a Kolmogorov-type diffusion and harder than $p=-2.3$ for an energyindependent diffusion, as shown in the left panels and right panels of Figure 10, respectively. Similar to the case of Geminga, given a Kolmogorov-type diffusion for the inner region, the positron flux produced by PSR B0656+14 contributes to $\leqslant 15 \%$ of the observed flux at energies below $400 \mathrm{GeV}$, with the spectrum rising too steeply compared with the data. However, if an energyindependent diffusion is assumed, PSR B0656+14 is allowed to contribute up to $30 \%$ of the observed positron flux at $100 \mathrm{GeV}$ and up to $50 \%$ of the positron flux at $400 \mathrm{GeV}$ by the current data.
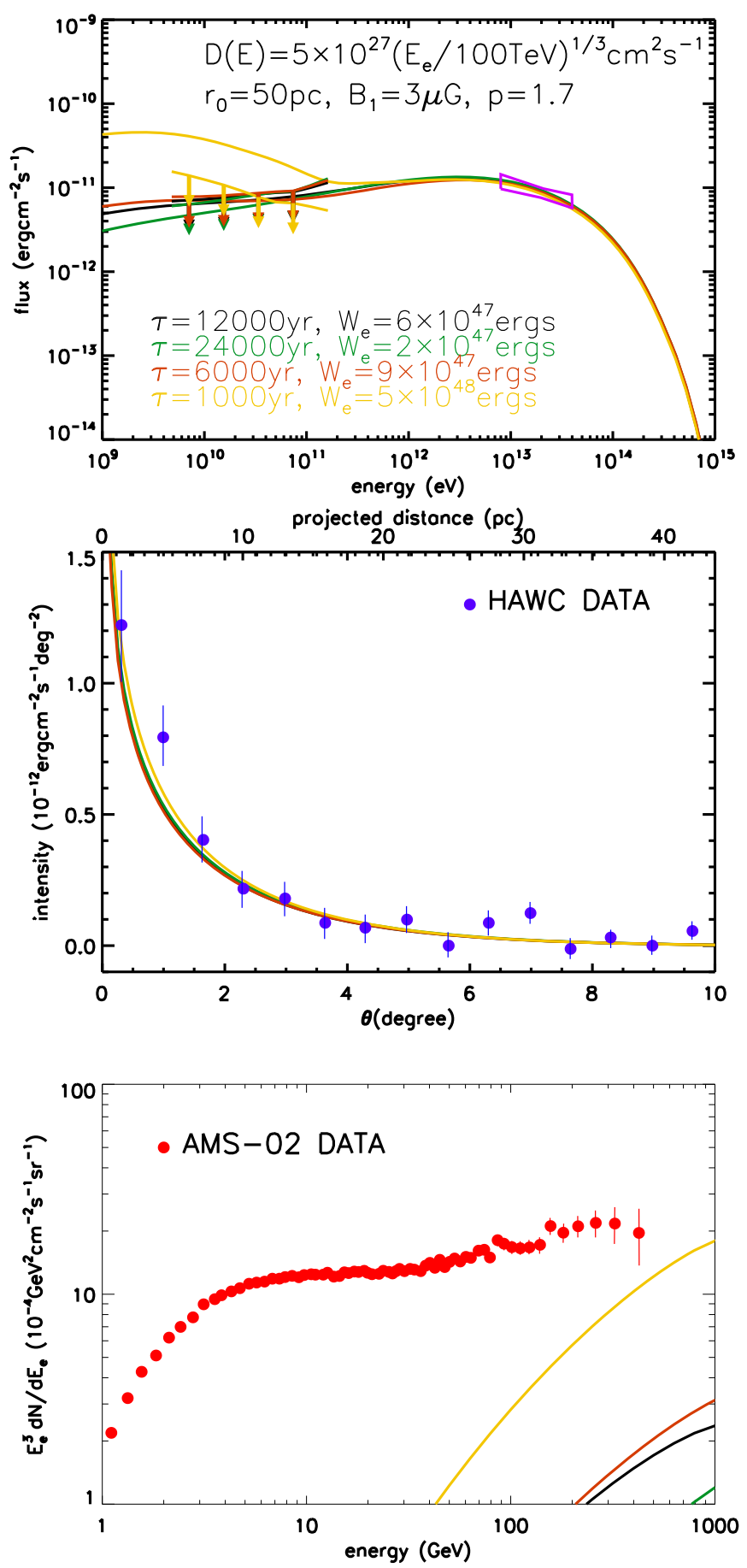

Figure 9. The effect of the spin-down timescale $\tau$. The employed parameters are labeled in the figure. See Section 4.2 for additional details.

\section{Summary}

To summarize, we have shown that Fermi-LAT's observations of the TeV nebulae of Geminga and PSR B0656+14 provide more direct constraints on the positron density at $\mathrm{GeV}$ energies in the vicinity of the pulsars than the TeV observations, and hence on their contribution to the measured positron excess at Earth. We conclude that two nearby pulsars are disfavored as the dominant sources of the positron excess at $\sim 50-500 \mathrm{GeV}$ in the two-zone diffusion models with the usual Kolmogorov-type 

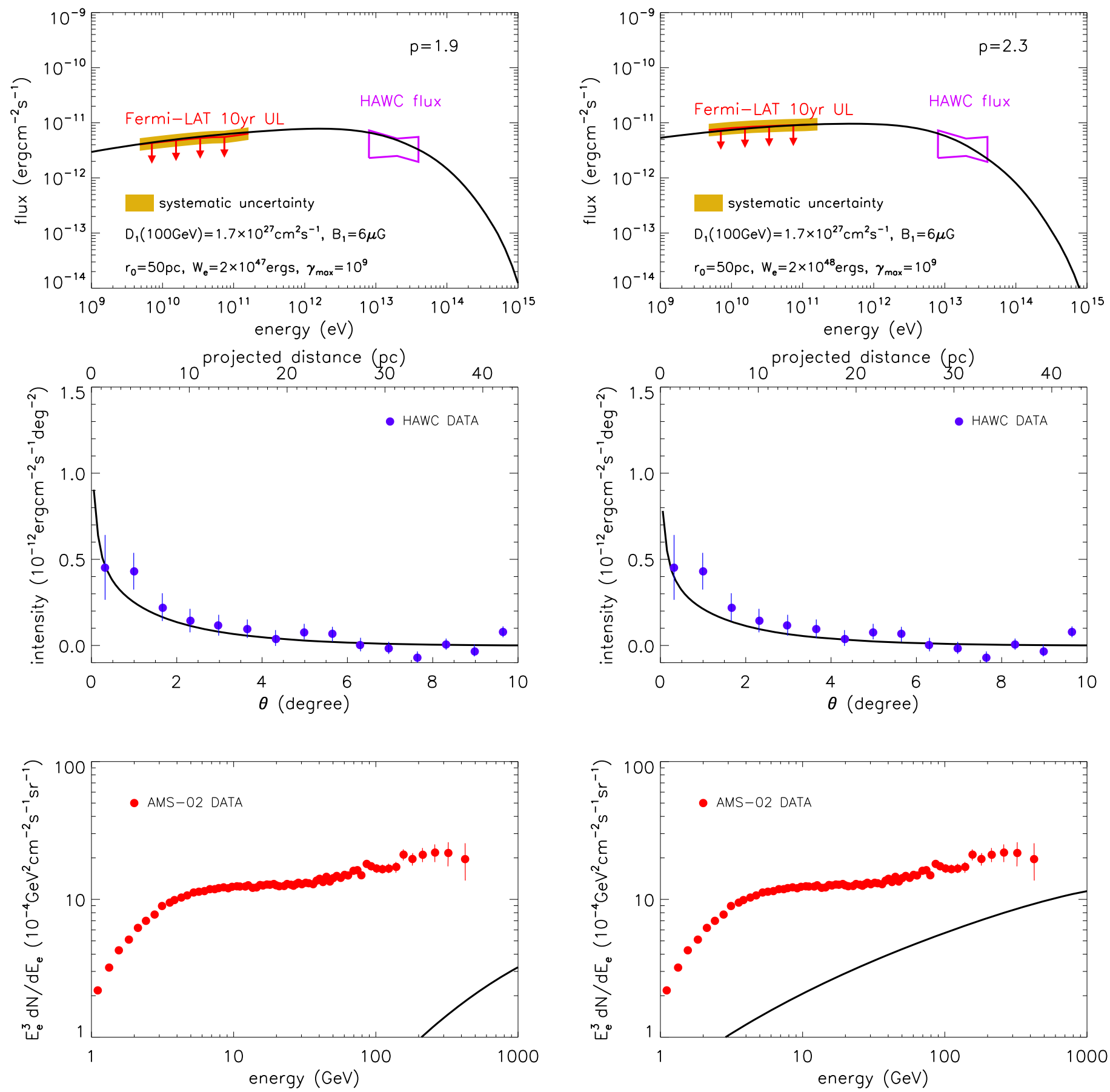

Figure 10. Top panel: comparison between the expected flux and the observation within $25^{\circ}$ of PSR B0656+14. Middle panel: the fit of the SBP data of PSR B0656 +14 measured by HAWC. Bottom panel: comparison between the expected positron flux produced by PSR B0656+14 and the observed flux at Earth. The left panels are under the assumption of a Kolmogorov-type diffusion, while the right panels are under the assumption of an energy-independent diffusion.

diffusion coefficient. In this case, the positron excess must be largely contributed by other sources, such as other pulsars, other types of nearby cosmic accelerators such as supernova remnants (Fujita et al. 2009) and microquasars (Gupta \& Torres 2014), or even the annihilation or decay of dark matter particles (e.g., Bergström et al. 2009; Yin et al. 2009; Cholis \& Hooper 2013; Silk 2014). However, for an energy-independent diffusion in the $\mathrm{TeV}$ nebulae, a dominant contribution of the positron excess by them cannot be ruled out by the current data.

This work has made use of data and software provided by the Fermi Science Support Center. X.Y.W. is supported by the
National Key R \& D program of China under the grant 2018YFA0404200 and the NSFC grants 11625312 and 11851304.

\section{Appendix \\ Systematic Uncertainty}

To assess the robustness of our results listed in Table 1 of the main text, we perform a number of systematic checks. Taking two templates (the Diffusion 1 and Diffusion 2 templates) as examples, we quantify the variation of the upper limits and the $\mathrm{TS}$ values. We first investigate the uncertainty from Galactic interstellar diffuse emission (IEM) background modeling. IEM 

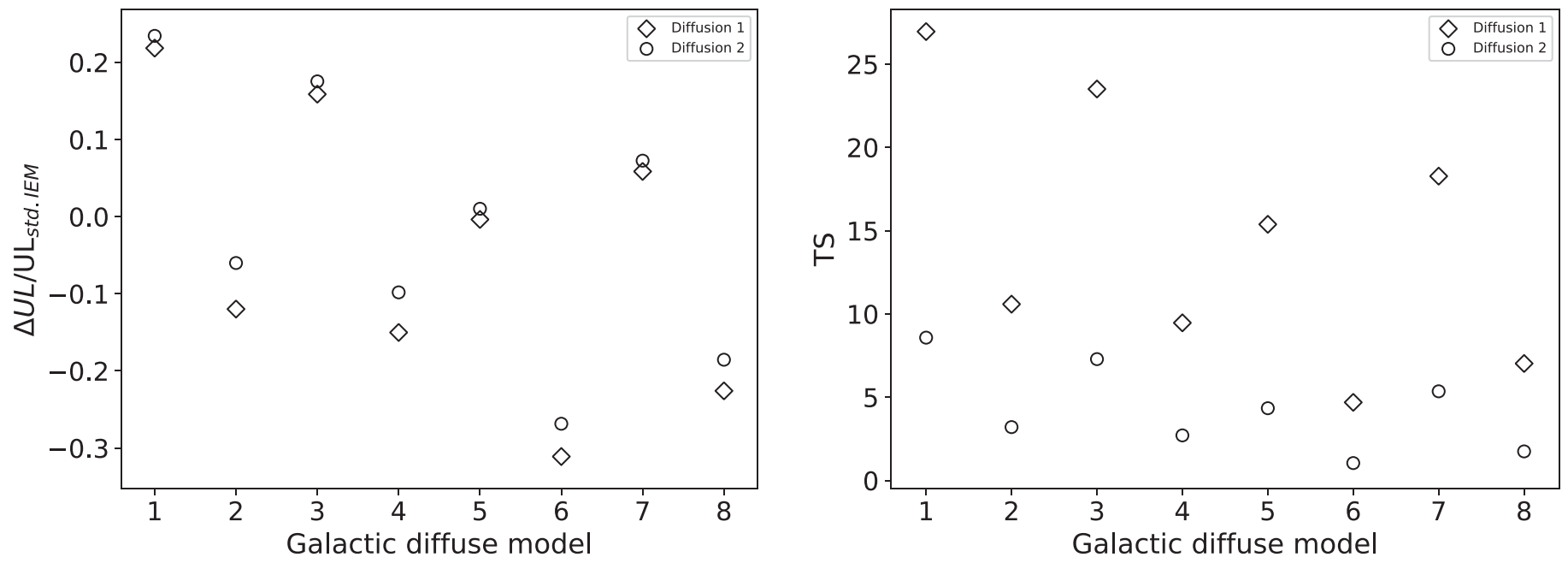

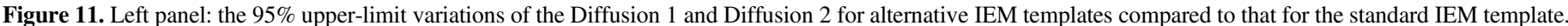
Right panel: the TS values for the Diffusion 1 and Diffusion 2 templates for each alternative IEM templates.

is produced through interactions of high-energy CR hadrons and leptons with interstellar gas via nucleon-nucleon inelastic collisions and electron bremsstrahlung, and with low-energy radiation fields via IC scattering. The standard IEM template released by the Fermi-LAT collaboration is created using the assumption that energetic CRs uniformly penetrate all gas phases of the ISM. Under this assumption, the IEM intensities at any energy can be modeled as a linear combination of templates of gas column densities, an IC intensity map, the templates that partially accounts for the emission from Loop I and the Fermi bubbles. The linear combination coefficients can be determined from a fit to gamma-ray data (Acero et al. 2016a). To explore the uncertainties related to this standard modeling of interstellar emission, we generated eight alternative IEMs to probe key sources of systematics by varying a few important input parameters to GALPROP (see Table 3). In addition, we adopt a different model-building strategy from the standard IEM. Starting from the work in Ackermann et al. (2012a), which provides 128 sets of intensity map associated with $\mathrm{H}_{\mathrm{I}}, \mathrm{H}_{\mathrm{II}}, \mathrm{CO}$, and IC, we select eight intensity map sets corresponding to the model parameters listed in Table 3. The intensity maps associated with gas are binned into four Galactocentric rings $(0-4 \mathrm{kpc}, 4-8 \mathrm{kpc}, \quad 8-10 \mathrm{kpc}$, and $10-20 \mathrm{kpc})$. We fit all model intensity maps simultaneously with an isotropic component, Loop I, Fermi bubbles, and 3FGL sources (Acero et al. 2015) to $8 \mathrm{yr}$ of Fermi-LAT data in order to determine the linear combination coefficients. We use eight alternative IEM templates to replace the standard one and perform the likelihood analysis to estimate uncertainties in the upper limits and TS values. We emphasize that these eight templates do not reflect the complete uncertainty of the IEMs, but the resulting uncertainty should be a reasonable indicator of the systematic error due to the mismodeling of the IEM background. We find that varying the IEM models increases the upper limits by at most 23\%, as shown in Figure 11.

Moreover, we study the systematic uncertainty caused by different selections of the ROI. Using the $40^{\circ} \times 40^{\circ}$ square ROI paralleling to R.A. and decl. (dubbed as alt. ROI), we find that the TS value of the Diffusion 1 template decreases significantly and the upper limits also decrease by $\sim 20 \%$. We further check the results using a smaller ROI $\left(30^{\circ} \times 30^{\circ}\right)$ and a larger ROI $\left(50^{\circ} \times 50^{\circ}\right)$, respectively, and find that the results
Table 3

Alternative Models for Galactic Diffuse Emission

\begin{tabular}{lccc}
\hline \hline Model Number & CR Source Distribution & $\begin{array}{c}\text { Halo Size } \\
(\mathrm{kpc})\end{array}$ & $\begin{array}{c}\text { Spin Temperature } \\
(\mathrm{K})\end{array}$ \\
\hline 1 & Lorimer & 4 & 150 \\
2 & Lorimer & 4 & $10^{5}$ \\
3 & Lorimer & 10 & 150 \\
4 & Lorimer & 10 & $10^{5}$ \\
5 & SNR & 4 & 150 \\
6 & SNR & 4 & $10^{5}$ \\
7 & SNR & 10 & 150 \\
8 & SNR & 10 & $10^{5}$ \\
\hline
\end{tabular}

Note. Overview of the alternative IEM models used for assessing the systematic uncertainties. We chose to vary the three most important input parameters that were found in scanning the parameter space in Ackermann et al. (2012a). Note that we use radial boundaries of $20 \mathrm{kpc}$ and apply a magnitude cut to the $E(B-V)$ maps at 5 . For the CR source distribution, we adopted the distribution of supernova remnants (SNRs) according to Case \& Bhattacharya (1998) and of pulsars according to Lorimer et al. (2006).

derived from the smaller ROI are close to that of alt.ROI analysis, and the results from the larger ROI are close to that of the standard analysis. We also test the systematic uncertainty using different event class data, which can be considered as an indicator for checking the impact of isotropy emission component. For the upper-limit estimation, the systematic uncertainty caused by the uncertainty in the effective area is also included.

We summarize the above systematic uncertainties in Table 2. It is worth noting that the TS values of the Diffusion 1 template ranges from 5 to 33 in our systematic checks, which may indicate that the detection significance suffers from systematic uncertainties. To be conservative, we here give the upper limits on the GeV flux, which can be used to constrain the contribution to the positron flux by Geminga. The maximum of the total systematic uncertainty for the Diffusion 2 template is $32 \%$. For simplicity, we use this maximum value as an approximation for the total systematic uncertainty of all other diffusion templates as well. 


\section{References}

Abdo, A. A., Allen, B. T., Aune, T., et al. 2009, ApJL, 700, L127 Abeysekara, A. U., Albert, A., Alfaro, R., et al. 2017a, Sci, 358, 911 Abeysekara, A. U., Albert, A., Alfaro, R., et al. 2017b, ApJ, 843, 40 Acero, F., Ackermann, M., Ajello, M., et al. 2015, ApJS, 218, 23 Acero, F., Ackermann, M., Ajello, M., et al. 2016a, ApJS, 224, 8 Acero, F., Ackermann, M., Ajello, M., et al. 2016b, ApJS, 223, 26 Ackermann, M., Ajello, M., Albert, A., et al. 2012a, ApJ, 750, 3 Ackermann, M., Ajello, M., Allafort, A., et al. 2012b, ApJS, 203, 4 Ackermann, M., Ajello, M., Atwood, W. B., et al. 2012c, PhRvL, 108, 011103 Ackermann, A., Ajello, M., Baldini, L., et al. 2011, ApJ, 726, 17 Adriani, O., Barbarino, G. C., Bazilevskaya, G. A., et al. 2009, Natur, 458, 607 Aguilar, M., Alberti, G., Alpat, B., et al. (AMS Collaboration) 2013, PhRvL, 110,141102

Aguilar, M., Aisa, D., Alpat, B., et al. 2014, PhRvL, 113, 221102 Aharonian, F., Atoyan, A. M., \& Völk, H. J. 1995, A\&A, 294, L41 Ajello, M., Atwood, W. B., Baldini, L., et al. 2017, ApJS, 232, 18 Bergström, L., Bringmann, T., \& EdsjÖ, J. 2009, PhRvD, 78, 103520 Case, G. L., \& Bhattacharya, D. 1998, ApJ, 504, 761

Cholis, I., \& Hooper, D. 2013, PhRvD, 88, 023013
Evoli, C., Linden, T., \& Morlino, G. 2018, PhRvD, 98, 063017

Fang, K., Bi, X.-J., Yin, P.-F., \& Yuan, Q. 2018, ApJ, 863, 30

Fujita, Y., Kohri, K., Yamazaki, R., \& Ioka, K. 2009, PhRvD, 80, 063003

Gupta, N., \& Torres, D. F. 2014, MNRAS, 441, 3122

Hooper, D., Blasi, P., \& Serpico, P. D. 2009, JCAP, 01, 025

Hooper, D., Cholis, I., Linden, T., \& Fang, K. 2017, PhRvD, 96, 103013

Hooper, D., \& Linden, T. 2018, PhRvD, 98, 083009

Joshi, J. C., \& Razzaque, S. 2017, JCAP, 09, 029

Lorimer, D. R., Faulkner, A. J., Lyne, A. G., et al. 2006, MNRAS, 372, 777

Moderski, R., Sikora, M., Coppi, P. S., \& Aharonian, F. 2005, MNRAS, 363,954

Moskalenko, I. V., \& Strong, A. W. 1998, ApJ, 493, 694

Profumo, S. 2011, CEJPh, 10, 1

Profumo, S., Reynoso-Cordova, J., Kaaz, N., \& Silverman, M. 2018, PhRvD, 97, 123008

Silk, J. 2014, PhRvD, 89, 063539

Tang, X. P., \& Piran, T. 2019, MNRAS, 484, 3491

Yin, P. F., Yu, Z. H., Yuan, Q., \& Bi, X. J. 2013, PhRvD, 88, 023001

Yin, P. F., Yuan, Q., Liu, J., et al. 2009, PhRvD, 79, 023512

Yuskel, H., Kistler, M. D., \& Stanev, T. 2009, PhRvL, 103, 051101 